\title{
Metabolomics analysis identifies different metabotypes of asthma severity
}

\author{
Stacey N. Reinke ${ }^{1}$, Héctor Gallart-Ayala ${ }^{1}$, Cristina Gómez ${ }^{1,2}$, Antonio Checa ${ }^{1,2}$, \\ Alexander Fauland ${ }^{1,2}$, Shama Naz ${ }^{1}$, Muhammad Anas Kamleh¹, \\ Ratko Djukanović ${ }^{3,4}$, Timothy S.C. Hinks ${ }^{3,4,5}$ and Craig E. Wheelock ${ }^{1}$
}

Affiliations: ${ }^{1}$ Division of Physiological Chemistry 2, Dept of Medical Biochemistry and Biophysics, Karolinska Institutet, Stockholm, Sweden. ${ }^{2}$ Institute of Environmental Medicine and the Centre for Allergy Research, Karolinska Institutet, Stockholm, Sweden. ${ }^{3}$ Clinical and Experimental Sciences, Faculty of Medicine, University of Southampton, Southampton, UK. ${ }^{4}$ Southampton NIHR Respiratory Biomedical Research Unit, University Hospital Southampton, Southampton, UK. ${ }^{5}$ Dept for Microbiology and Immunology, The Peter Doherty Institute for Infection and Immunity, University of Melbourne, Melbourne, Australia.

Correspondence: Craig E. Wheelock, Division of Physiological Chemistry 2, Dept of Medical Biochemistry and Biophysics, Karolinska Institutet, SE-171 77 Stockholm, Sweden. E-mail: craig.wheelockaki.se

@ERSpublications

Mild asthma is metabolically distinct from both moderate and severe asthma, and steroid treatment affects metabolism http://ow.ly/EHo7306DwmN

Cite this article as: Reinke SN, Gallart-Ayala H, Gómez C, et al. Metabolomics analysis identifies different metabotypes of asthma severity. Eur Respir J 2017; 49: 1601740 [https://doi.org/10.1183/13993003.017402016].

ABSTRACT In this study, we sought to determine whether asthma has a metabolic profile and whether this profile is related to disease severity.

We characterised the serum from 22 healthy individuals and 54 asthmatics (12 mild, 20 moderate, 22 severe) using liquid chromatography-high-resolution mass spectrometry-based metabolomics. Selected metabolites were confirmed by targeted mass spectrometry assays of eicosanoids, sphingolipids and free fatty acids.

We conclusively identified 66 metabolites; 15 were significantly altered with asthma $(p \leqslant 0.05)$. Levels of dehydroepiandrosterone sulfate, cortisone, cortisol, prolylhydroxyproline, pipecolate and N-palmitoyltaurine correlated significantly $(\mathrm{p}<0.05)$ with inhaled corticosteroid dose, and were further shifted in individuals treated with oral corticosteroids. Oleoylethanolamide increased with asthma severity independently of steroid treatment $(\mathrm{p}<0.001)$. Multivariate analysis revealed two patterns: 1) a mean difference between controls and patients with mild asthma ( $\mathrm{p}=0.025)$, and 2) a mean difference between patients with severe asthma and all other groups $\left(\mathrm{p}=1.7 \times 10^{-4}\right)$. Metabolic shifts in mild asthma, relative to controls, were associated with exogenous metabolites (e.g. dietary lipids), while those in moderate and severe asthma (e.g. oleoylethanolamide, sphingosine-1-phosphate, N-palmitoyltaurine) were postulated to be involved in activating the transient receptor potential vanilloid type 1 (TRPV1) receptor, driving TRPV1-dependent pathogenesis in asthma.

Our findings suggest that asthma is characterised by a modest systemic metabolic shift in a disease severity-dependent manner, and that steroid treatment significantly affects metabolism.

This article has supplementary material available from erj.ersjournals.com

Received: Sept 012016 | Accepted after revision: Nov 232016

Copyright OERS 2017. This version is distributed under the terms of the Creative Commons Attribution Licence 4.0. 


\section{Introduction}

Asthma is a common chronic respiratory disease driven by heterogeneous inflammatory mechanisms that result in multiple clinical phenotypes and inflammatory endotypes [1, 2], with inflammation, oxidative stress and tissue damage/remodelling playing integral roles [3]. Asthma presents clinically with a range of severity, from mild disease that is well-controlled with intermittent short-acting $\beta$-agonists, to severe disease with persistent symptoms despite long-term treatment, including short- and long-acting $\beta$-agonists, leukotriene receptor antagonists, corticosteroids and/or novel biologics [4]. The underlying disease mechanisms, as they relate to disease severity, have been the subject of significant research, but remain poorly understood $[2,3]$.

Metabolomics is the systematic study of low molecular weight (bio)chemicals in a given biological system [5]. Metabolites play a central role in homeostasis and disease, contributing to processes such as redox balance, oxidative stress, signalling, apoptosis and inflammation. The metabolic state represents the convergence of gene expression and environmental exposure, and can provide an informative measure of multifactorial diseases. Although metabolomics offers a powerful approach to biomarker discovery and elucidating disease mechanisms, inter- and intra-study irreproducibility often arises from technical inconsistency, absent metabolite inclusion criteria, inappropriate or overtrained chemometric analyses and poor reporting standards [6, 7]. Patient characterisation in metabolomic studies to date has been diverse, with some studies classifying asthma as a single disease phenotype [8-10], or comparing stable and unstable [11] or severe and non-severe forms [12]. Additionally, analytical reproducibility measures and metabolite inclusion criteria have often not been reported.

The primary aim of this study was to determine whether there is a metabolic signature of asthma that would allow us to characterise the metabotype of asthma. The secondary aim was to determine if this signature is related to disease severity. We applied liquid chromatography-high-resolution mass spectrometry (LC-HRMS) to characterise the serum metabolic profiles from healthy controls and from asthmatic patients recruited from a previous study [13] classified by clinical activity as having mild, moderate or severe disease. Following multivariate modelling, the concentrations of selected metabolites were confirmed with targeted mass spectrometry assays of eicosanoids, sphingolipids and free fatty acids.

\section{Methods}

Clinical cohort and sample collection

Seventy-eight participants (18-70 years old) were enrolled. The results from two patients with severe asthma were identified as outliers and removed from further analysis (supplementary figure E1), leaving 22 healthy non-atopic controls and 54 asthmatic individuals (table 1). Asthmatic individuals were stratified according to patient-reported use and a review of clinical records [1] as follows: 12 patients with mild asthma, with intermittent symptoms treated with short acting $\beta_{2}$-agonists alone; 20 patients with moderate asthma, with frequent symptoms treated with inhaled corticosteroids (ICS); and 22 patients with severe asthma, with persistent symptoms despite receiving high-dose ICS $(\mathrm{n}=22)$ and oral corticosteroids (OCS) $(n=5)$. All participants were enrolled from the National Institute for Health Research Southampton Respiratory Biomedical Research Unit and University Hospital Southampton outpatient clinics. The National Research Ethics Service Committee South Central - Southampton B ethics committee (UK; Ref 10/H0504/2) approved this study and all participants provided informed written consent. Clinical classification (supplementary table E1) and enrolment criteria have previously been described [13, 14]. Blood samples were allowed to clot at room temperature for $30 \mathrm{~min}$, then centrifuged at $1650 \times g$ for $10 \mathrm{~min}$ at $4^{\circ} \mathrm{C}$, and $1 \mathrm{~mL}$ of serum was stored at $-80^{\circ} \mathrm{C}$ until analysis.

\section{Liquid chromatography-mass spectrometry}

Details of the mass spectrometry-based analyses for metabolomics are described in the supplementary methods. Briefly, $50 \mu \mathrm{L}$ of serum were precipitated for reversed-phase or hydrophilic interaction chromatography. Samples were analysed using an Ultimate 3000 UHPLC system coupled to a Thermo

Support statement: S.N. Reinke was supported by a Canadian Institutes of Health Research Fellowship (MFE-135481). A. Fauland was funded by the Karin and Sten Mörstedt Initiative on Anaphylaxis. T.S.C. Hinks was supported by fellowships from the Wellcome Trust $(088365 / \mathrm{z} / 09 / \mathrm{z}, 104553 / \mathrm{z} / 14 / \mathrm{z})$ and the Academy of Medical Sciences. C.E. Wheelock was supported by the Swedish Heart Lung Foundation (HLF 20150640). We acknowledge the support of the Swedish Heart Lung Foundation (HLF 20140469), the Karolinska Institutet and AstraZeneca Joint Research Programme in Translational Science (ChAMP Project), and the National Institute for Health Research (NIHR), through the Primary Care Research Network and through an Academic Clinical Fellowship awarded to T.S.C. Hinks. Infrastructure support was funded by the NIHR Southampton Respiratory Biomedical Research Unit. Funding information for this article has been deposited with the Open Funder Registry.

Conflict of interest: Disclosures can be found alongside this article at erj.ersjournals.com 
TABLE 1 Clinical information

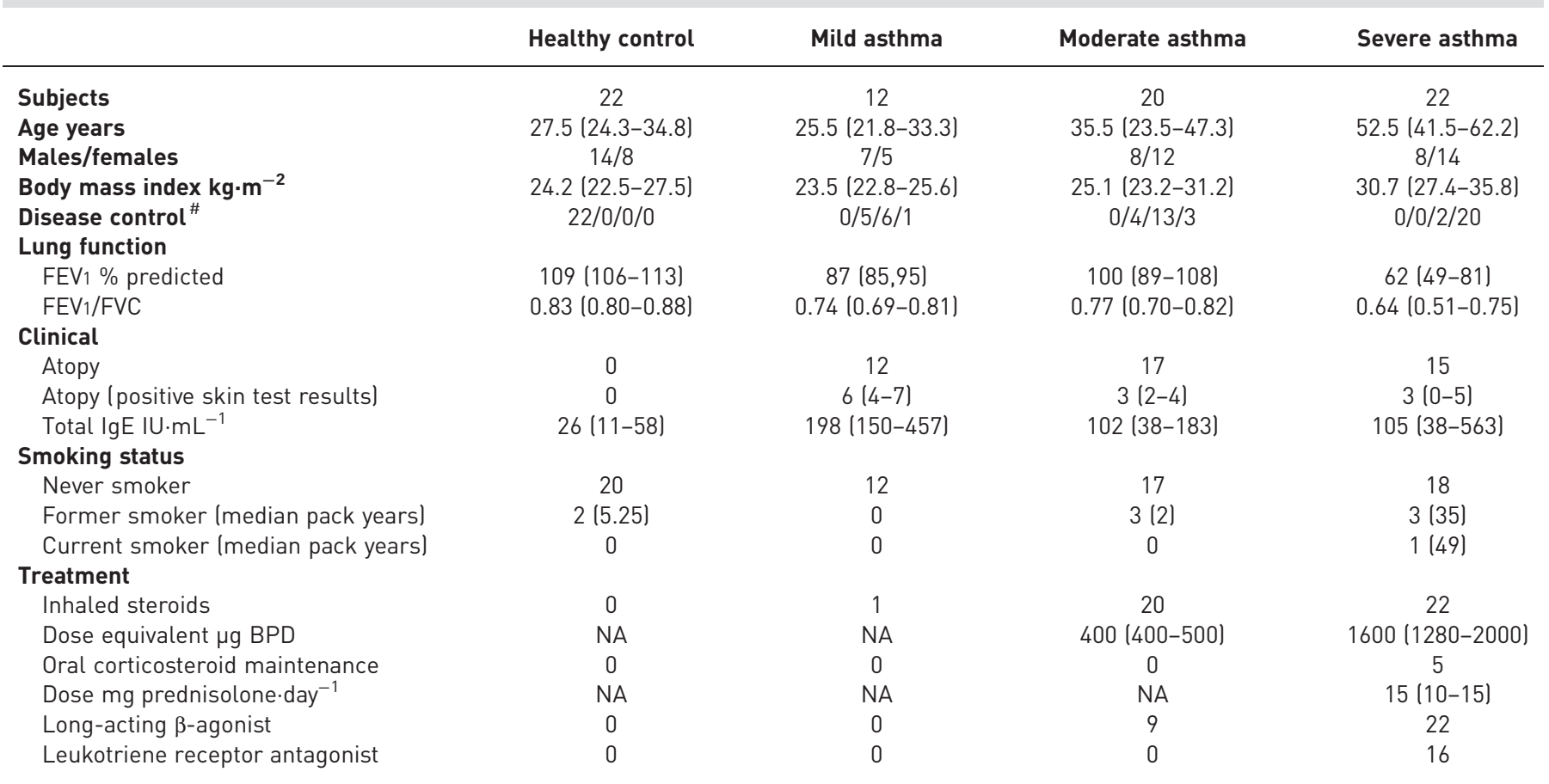

Data are presented as median (interquartile range) or number. FEV1: forced expiratory volume in 1 s; FVC: forced vital capacity; BPD: beclomethasone dipropionate equivalent; NA: not applicable. "Global Initiative for Asthma [1] level of control, presented as number of subjects with score of $0 / 1 / 2 / 3$, where $0=$ no asthma, $1=$ controlled, $2=$ partly controlled, $3=$ uncontrolled.

Q-Exactive (Orbitrap) mass spectrometer (Thermo Fisher Scientific, San Jose, CA, USA). Peak deconvolution was performed using the TraceFinder software v3.1 (Thermo Fisher Scientific). Metabolites were identified by matching accurate mass and retention time to an in-house chemical reference database of 600 metabolites, with confirmation determined by comparing tandem mass spectrometry (MS/MS) fragmentation patterns and product ion abundance to those of chemical standards. All metabolites included in statistical analyses were confirmed by MS/MS or by a targeted assay.

\section{Data processing}

Before data cleaning, systematic experimental variation was corrected using the Quality Control-Robust Spline Correction (QC-RSC) algorithm $[15,16]$. The number of missing values was determined for each metabolite. The relative standard deviation (RSD) was calculated for the pooled QC injections (RSDQC) and the sample injections (RSDSample); the ratio of RSDSample:RSDQC was also determined. Metabolites with $>20 \%$ missing values, RSDQC $>25 \%$ or an RSD ratio $<1.5$ were deemed not suitably reproducible and excluded from the statistical analysis. Principal component analysis was performed to identify sample outliers; two samples were excluded from further analysis (supplementary figure E1).

\section{Statistical analysis}

Data, expressed as relative abundance, were not normally distributed (Lilliefors test); therefore, non-parametric tests were used for all univariate analyses. Median fold-change and confidence intervals were estimated using bootstrap resampling with 500 iterations [17]. The null hypothesis that each metabolite distribution was identical across all clinical groups was tested using the Kruskal-Wallis test, with correction for multiple comparisons according to Benjamini and Hochberg (q-value). Correlations between metabolite abundances and ICS dose were tested using pairwise Spearman's rank correlation coefficients (r).

Pairwise Spearman's rank correlation coefficients ( $r$ ) were also calculated to assess the strength and direction of linear relationships between metabolites and disease severity. The resulting correlation matrix is presented in the form of a spring-embedded correlation plot $[6,18]$. A network of "nodes" and "spring-edges" was constructed; nodes represent each of the tested metabolites $(p<0.1)$ and edges are proportional to the correlation coefficient between two connected nodes. Node size is proportional to the significance of that metabolite; the larger nodes represent lower p-values. Edges represent positive correlation coefficients significant at $\mathrm{p}<0.05$. The resulting plot can be interpreted as a simple multivariate analysis, whereby nodes 
clustering together are considered highly correlated. Additional information on generating this plot can be found in the original publications $[6,18]$.

Principal component-canonical variate analysis (PC-CVA) [19] was performed to visualise multifactorial and correlated discrimination between the clinical groups. Prior to multivariate analysis, data were log-transformed and scaled to unit variance, and missing data points were input using $\mathrm{k}$-nearest neighbours $(\mathrm{k}=3)$ [20]. The number of PCs to be projected into CVA space was determined using $\mathrm{k}$-fold cross-validation (supplementary figure E2). Bootstrap resampling/remodelling were performed to determine metabolites that significantly contributed to the CVA model $[17,21]$.

Statistical analyses were performed using Stata v.12 (StataCorp LP, College Station, TX, USA) or the MATLAB scripting language (Mathworks, Natick, MA, USA). The spring plot was created using the "neato" virtual physics models in the Graphviz software (www.graphviz.org).

\section{Targeted assays}

Three mass spectrometry-based targeted methods were additionally performed to confirm selected metabolomics results, including quantification of a panel of eicosanoids, sphingolipids and free fatty acids. For more details, see the supplementary methods.

\section{Results}

\section{Metabolomic analysis}

LC-HRMS analysis of protein-free serum resulted in the reproducible detection of 66 MS/MS identity-confirmed metabolites, including amino acids, biogenic amines, excretion metabolites, fatty acids, lipid mediators, steroids, bile acids and purine/pyrimidine metabolites (supplementary table E2). An assessment of quality control samples revealed an average relative standard deviation (QCRSD) of $11 \%$.

With a significance level of $\mathrm{p}<0.05$ for the primary comparison between asthma and health, 15 metabolites were found to be significantly dysregulated in asthma (table 2). The relative abundances of oleoylethanolamide (OEA), 22-hydroxycholesterol, $\alpha$-linolenic acid and sphingosine-1-phosphate (S1P(d18:1)) were increased in all

TABLE 2 Selected metabolomics data

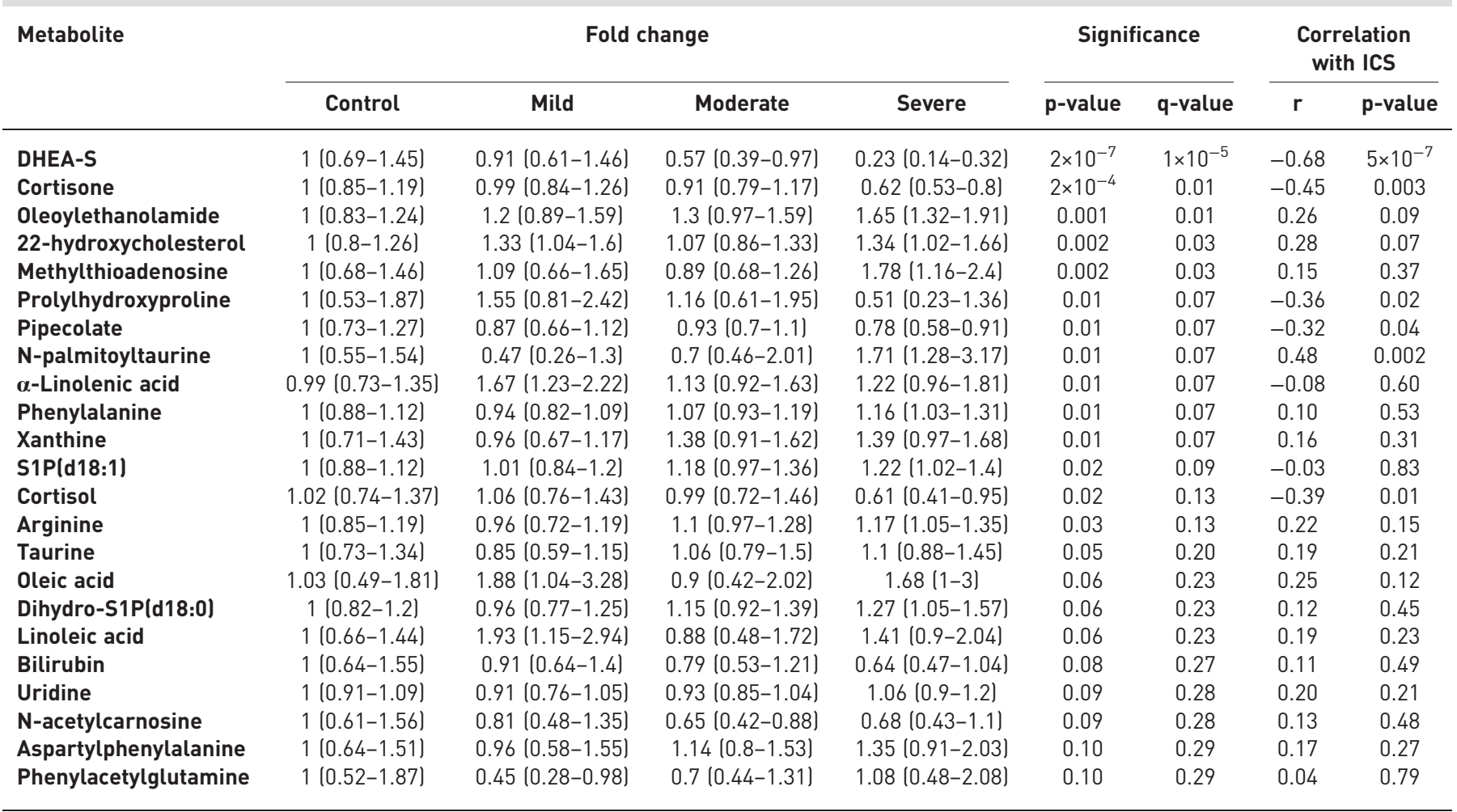

Data are presented as the fold-change of the median $(95 \% \mathrm{Cl}$ ). Displayed data were selected based upon $p<0.1$. The full list of all detected metabolites is provided in supplementary table E3. Median fold change and confidence intervals were estimated using 500 iterations of bootstrap resampling. ICS: inhaled corticosteroids; S1P: sphingosine-1-phosphate. 
asthma subgroups. In the moderate and severe asthma groups, the levels of dehydroepiandrosterone sulfate (DHEA-S), cortisone and pipecolate were decreased, while levels of taurine, phenylalanine, xanthine and arginine were increased. Cortisol was decreased only in the patients with severe asthma. Methylthioadenosine was slightly decreased in moderate asthma and highly increased in severe asthma. The levels of taurine, phenylalanine, xanthine and arginine were decreased slightly in mild asthma. Of particular interest, prolylhydroxyproline (ProHyp) and N-palmitoyltaurine were increased in mild asthma, but decreased in severe asthma. Eight additional metabolites tended to be differentially abundant in asthmatic patients when compared to healthy controls at significance levels between $\mathrm{p}=0.05$ and 0.10 : oleic acid, dihydrosphingosine-1-phosphate $(\mathrm{dhS1P}(\mathrm{d} 18: 0))$, linoleic acid, bilirubin, uridine, N-acetylcarnosine, aspartylphenylalanine and phenylacetylglutamine. The relative abundances of all these metabolites shifted differentially between the study severity groups, with bilirubin and $\mathrm{N}$-acetylcarnosine decreasing across all groups.

Six metabolites correlated significantly $(\mathrm{p}<0.05)$ with the ICS dose that the asthmatic patients were on as regular treatment: DHEA-S, cortisone, ProHyp, pipecolate, N-palmitoyltaurine and cortisol. For all these metabolites, except for pipecolate, the change in median abundance (relative to healthy controls) was more pronounced in the five asthmatic patients on maintenance OCS than in those on ICS alone (figure 1a-c). Conversely, the median relative abundance of OEA (not significantly correlated with ICS dose) was not affected by OCS (figure 1d). No significant interaction was observed between ICS dose and metabolite levels (data not shown). An analysis of the ratios of metabolites affected by ICS/OCS did not provide stronger correlations (e.g. correlation of DHEA-S with ICS dose $\left(\mathrm{r}=-0.68, \mathrm{p}=5 \times 10^{-7}\right)$ versus correlation of OEA:DHEA-S with ICS dose $\left.\left(\mathrm{r}=0.62, \mathrm{p}=1 \times 10^{-5}\right)\right)$.

Spring-embedded correlation analysis (figure 2) identified two primary clusters of metabolites: one cluster was composed of metabolites that decreased with respect to asthma severity and correlated with DHEA-S, while the other comprised metabolites that increased with asthma severity; S1P(d18:1), OEA, N-palmitoyltaurine, 22-hydroxycholesterol and xanthine were central to numerous correlations within the
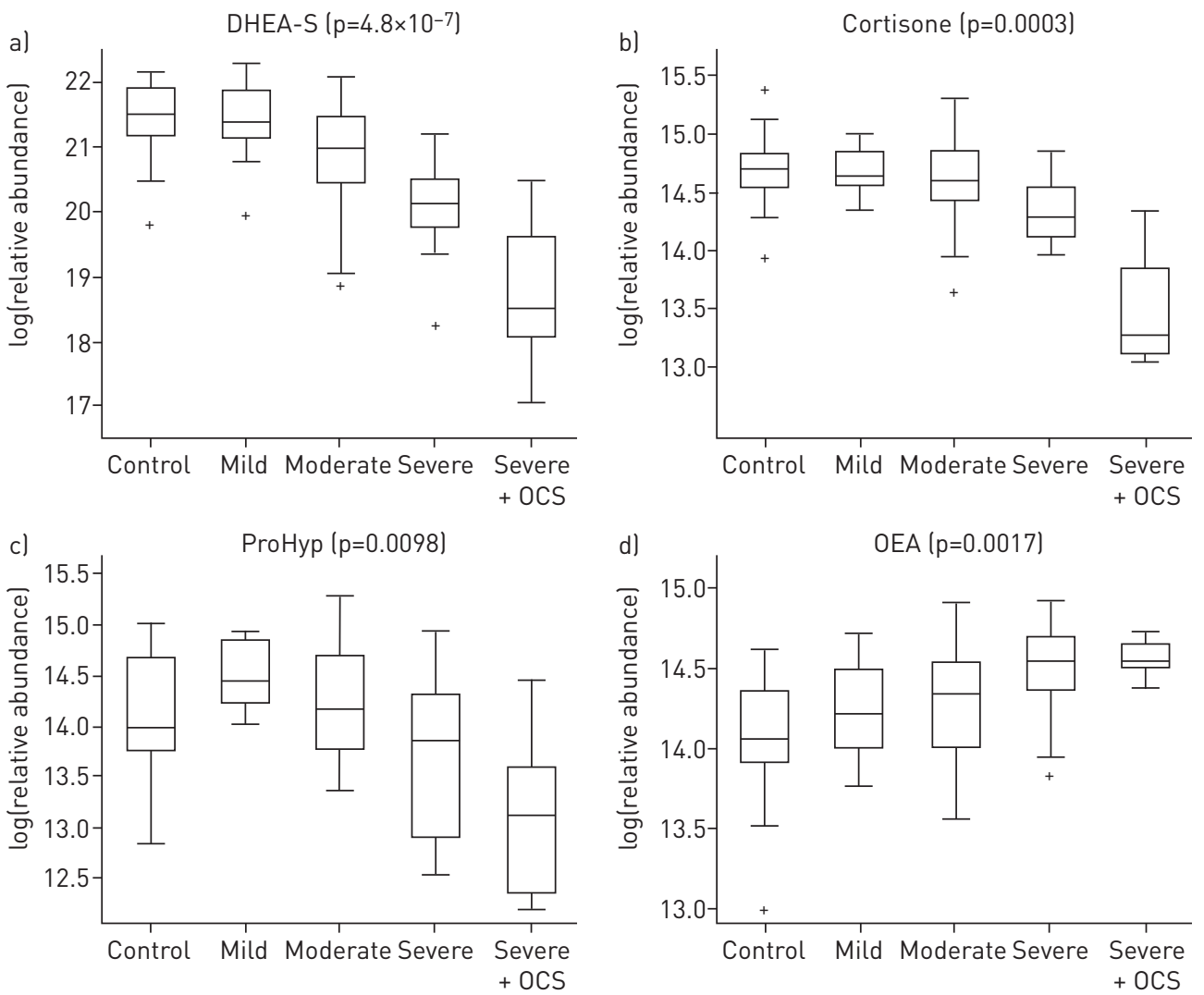

FIGURE 1 Association between oral corticosteroid (OCS) treatment and the relative abundance of selected metabolites. Severe asthma was classified according to treatment. Severe: treatment with inhaled corticosteroids only; Severe+OCS: treated also with oral corticosteroids. The line in the middle of each box equals the median value, the tops and bottoms of each box are the first and third quartile, respectively. The whiskers span from 1.5 times the interquartile range (IQR) above the third quartile to 1.5 times the IQR below the first quartile. Samples outside this range (crosses) are considered outliers. Kruskal-Wallis p-values are shown. DHEA-S: dehydroepiandrosterone sulfate; ProHyp: prolylhydroxyproline; OEA: oleoylethanolamide. 


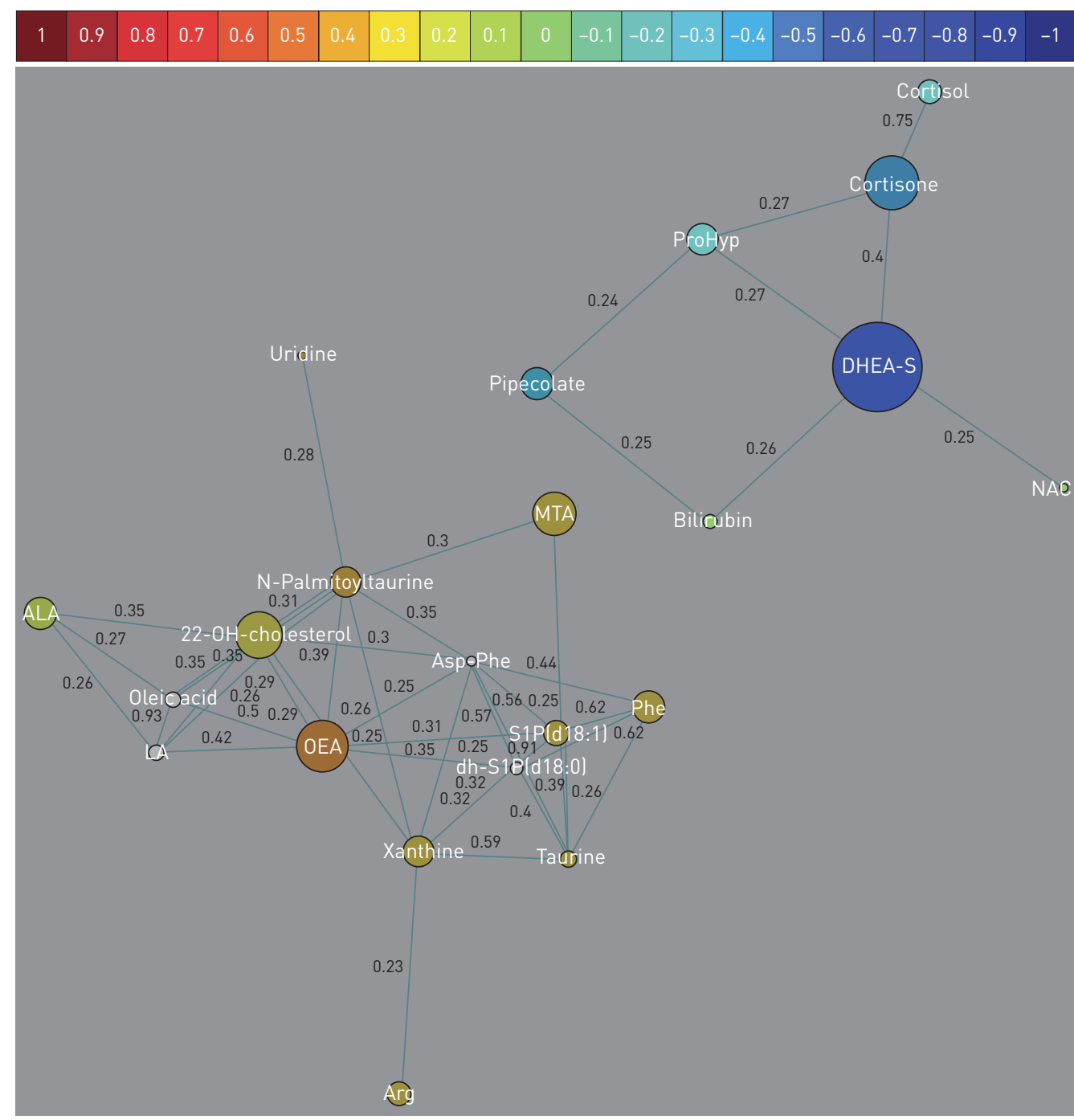

FIGURE 2 Spring-embedded plot illustrating the relationship between selected metabolites and disease severity. The size of the node is proportional to the significance of the relationship with disease severity (the larger the circle, the more significant the metabolite). The length of the line between the nodes (spring length) is proportional to the correlation strength (the shorter the length, the stronger the correlation with neighbouring metabolites). Node colour directly maps onto the correlation coefficient between the relative abundance of the metabolite and disease severity (see the colour bar above the figure: the intensity of the colours red and blue denote positive and negative correlations with disease severity, respectivelyl for the significant metabolites $(p<0.05)$. Nodes were coloured grey if their corresponding $p$-value was $0.05-0.10$. ALA: $\alpha$-linolenic acid; Arg: arginine; Asp-Phe: aspartylphenylalanine; dh-S1P(d18:0): dihydrosphingosine-1phosphate(d18:0); DHEA-S: dehydroepiandrosterone sulfate; LA, linoleic acid; MTA: methylthioadenosine; NAC: N-acetylcarnosine; OEA, oleoylethanolamide; Phe, phenylalanine; ProHyp, prolylhydroxyproline; S1P (d18:1), sphingosine-1-phosphate(d18:1).

cluster. However, overall the linear correlations between metabolite concentrations and disease severity were relatively weak; the strongest correlations were for DHEA-S $(r=-0.64, \mathrm{p}<0.001)$, OEA $(r=0.47, \mathrm{p}<0.001)$ and cortisone $(\mathrm{r}=-0.43, \mathrm{p}<0.001)$.

Results of the multivariate analysis using PC-CVA (figure $3 \mathrm{a}-\mathrm{c}$ ) were in agreement with the univariate and correlation analyses, revealing differential metabolic profiles for the three asthma severity groups. The first canonical variate (CV1, figure 3a) described a significant mean difference between the severe asthmatic and all other groups, including healthy controls $\left(\mathrm{p}=1.7 \times 10^{-4}\right)$. It also showed that moderate asthma has a heterogeneous metabolic profile, with mean values closer to, but significantly different $(\mathrm{p}=0.013)$ from, health than to severe asthma. The second canonical variate (CV2) described a significant mean separation $(p=0.025)$ between the mild asthma group and healthy control group. Bootstrap resampling/remodelling identified 22 metabolites that significantly contributed to the separation along CV1 (figure 3b), and 14 that significantly contributed to CV2 (figure 3c). Previously identified univariately altered metabolites (table 2) contributed 


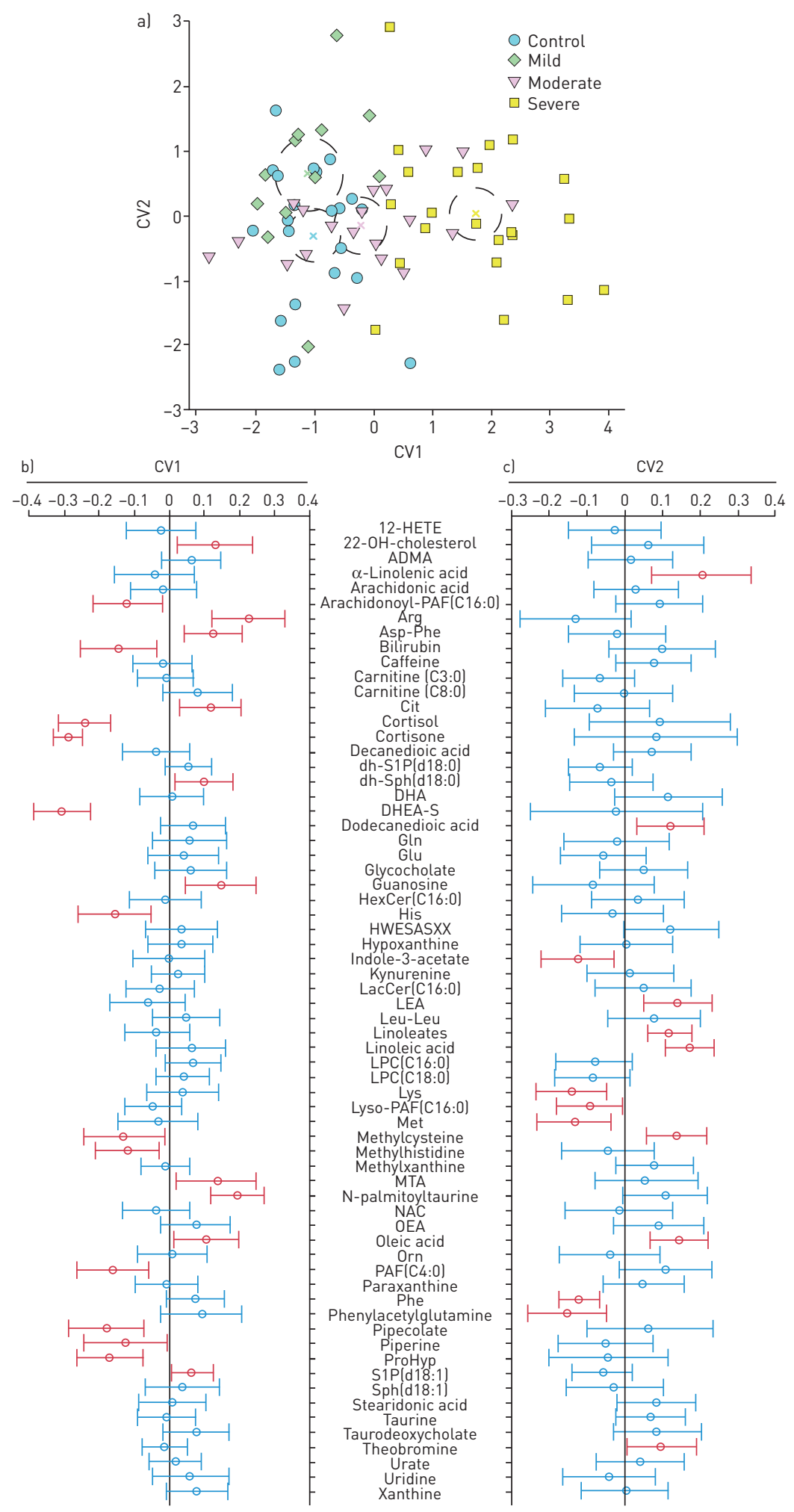

FIGURE 3 Principal component-canonical variate analysis (PC-CVA) of the relationship between metabolite levels and disease severity. a) Score plot labelled by clinical severity. Blue circles: healthy controls; green diamonds: mild asthma; pink triangles: moderate asthma; yellow squares: severe asthma; corresponding coloured X: mean of each group; dashed circles: 95\% confidence intervals of the mean of each group. b) Loadings illustrating contribution of metabolites along CV1 (left) and CV2 (right). Red: metabolites that significantly $(p<0.05)$ contribute to separation along the respective $C V$; blue, metabolites that do not significantly contribute to separation; circles: mean loading values; whiskers: $95 \%$ confidence intervals of the loadings. The PC-CVA model was constructed using eight principal components. Full metabolite names are listed in supplementary table E3. 
largely to separation along the CV1 axis (figure 3b). Metabolites uniquely contributing to differences between healthy controls and patients with mild asthma included phenylacetylglutamine, theobromine, $\alpha$-linolenic acid, linoleic acid and a heterogeneous peak of co-eluting compounds $(\mathrm{m} / z=295.2279 ; \Delta \mathrm{ppm}=$ $-0.94 \mathrm{ppm}$ ), corresponding to oxidation products of linoleic acid (hydroxyoctadecadienoic and/or epoxyoctadecenoic acids).

Additional, targeted assays were performed to confirm the observed trends in levels of 1) sphingolipids, 2) fatty acids and 3) eicosanoids and related analogues (oxylipins) (table 3). Based upon the observed increases in the levels of $\mathrm{S} 1 \mathrm{P}(\mathrm{d} 18: 1)$ and $\mathrm{dhS} 1 \mathrm{P}(\mathrm{d} 18: 0)$ with asthma, as well as the known relevance of sphingolipids to asthma pathogenesis [22, 23], we performed a targeted sphingolipid assay. Owing to the general poor chromatographic behaviour of fatty acids in the LC-HRMS metabolomics methods, in combination with the difficulty in performing MS/MS-based confirmation, we verified our findings using a targeted gas chromatography-mass spectrometry-based approach. Given the observed contribution of the linoleic acid oxidation products peak in our PC-CVA model $(\mathrm{m} / \mathrm{z}=295.2279)$, as well as the known importance of eicosanoids in asthma, we also performed a targeted eicosanoid assay. Full results are presented in supplementary tables E4-E6. Targeted analyses revealed similar trends for S1P(d18:1), dhS1P(d18:0) and $\alpha$-linolenic acid; the trends for linoleic acid and its oxidation products were less clear. The levels of ceramides and sphingomyelins were found to increase with asthma severity, whereas the hexosylceramides and lactosylceramdes did not change significantly (supplementary table E4). A number of free fatty acids increased in asthmatic patients, with levels generally highest in severe asthma (supplementary table E5). Within the eicosanoid panel, only cysteinyl leukotriene $\mathrm{E}_{4}$ increased with asthma severity $(\mathrm{p}<0.05)$ (supplementary table E6 and figure E3). Generally, there were no correlations with ICS, with the exception of ceramide(18:1), ceramide(16:0), sphingomyelin(12:0) and four free fatty acids (myristoic, palmitoleic, eicosenoic, and dihomo-gamma-linolenic acid), which were positively associated with the dose of ICS (table 3).

\section{Discussion}

This study has identified metabolic profiles of asthma that differentiate between mild, moderate and severe asthma. Of the 66 conclusively identified metabolites, 15 were significantly differentially regulated across the groups. All three endogenous steroid metabolites (cortisone, cortisol, DHEA-S) identified were significantly decreased in asthmatic patients on maintenance treatment with systemic steroids, consistent with current knowledge of steroid metabolism [24, 25]. However, DHEA-S was also suppressed in patients on ICS alone, thereby demonstrating a systemic effect of ICS treatment. Of particular interest is the observation that steroid-naïve patients with mild asthma exhibited a metabolic profile that was different from that of both healthy controls and patients with moderate or severe asthma (figure 3). Multivariate modelling identified eight metabolites that were increased and six that were decreased in patients with mild asthma when compared to healthy individuals, whereas 10 metabolites were increased and 12 were decreased in patients with moderate/severe asthma. Correlation analysis did not show a linear association between metabolite relative abundance and disease severity (figure 2). Together, these findings show that several circulating metabolites in asthma differ from those in healthy individuals and are related to disease severity, whereas some are influenced by steroid treatment.

In clinical terms, asthma is usually classified by overall severity as mild, moderate or severe [1]; our findings suggest that mild and moderate asthma are different phenotypes, as evidenced by their distinct metabolic profiles. In the current study, patients with mild asthma were characterised by infrequent symptoms, i.e. daytime symptoms less than once per day and nocturnal symptoms less than twice per month (supplementary table E1) that require treatment with short-acting $\beta$-agonists. Conversely, individuals with moderate asthma experienced more frequent symptoms, requiring ICS management and occasionally short-acting $\beta$-agonists. With respect to their metabolic profile, patients with moderate asthma shared the same PC-CVA axis as those with severe disease (CV1; figure 3). This indicates that the two groups share similar disease metabolic profiles even though moderate asthma responds well to therapeutic management and these patients more closely resemble the healthy individuals, although it is plausible that these groups could have been differentiated by other metabolites not included in our database. The persistence of metabolic abnormalities despite treatment with ICS and OCS suggests that there are distinct, relatively steroid-insensitive mechanisms that are associated with disease severity.

The metabolic shift in patients with mild asthma was primarily associated with exogenous metabolites (CV2; figure 3C), especially elevated levels of dietary lipids (e.g. linoleic acid, oleic acid, $\alpha$-linolenic acid) and linoleic acid oxidation products. Linoleic acid and its oxidation products are proposed as contributing to Th2 differentiation in asthma [26]; thus, the finding of their excessive abundance warrants further investigation in larger cohorts. Plant-derived methylcysteine [27] and theobromine [28], and the gut microbiome product phenylacetylglutamine [29] also contributed to the observed multivariate separation. Interestingly, decreased phenylacetylglutamine has been reported during asthma exacerbations [10] and 


\begin{tabular}{|c|c|c|c|c|c|c|c|c|}
\hline \multirow[t]{2}{*}{ Metabolite } & \multicolumn{4}{|c|}{ Fold change } & \multicolumn{2}{|c|}{ Significance } & \multicolumn{2}{|c|}{ Correlation with ICS } \\
\hline & Control & Mild & Moderate & Severe & p-value & q-value & $\mathbf{r}$ & p-value \\
\hline \multicolumn{9}{|l|}{ Sphingolipids } \\
\hline Ceramide(C18:0) & $1(0.78-1.27)$ & $1.12(0.85-1.38)$ & $1.21(0.71-1.55)$ & $1.56(1.2-2.03)$ & $4 \times 10^{-4}$ & 0.01 & 0.22 & 0.14 \\
\hline Sphingomyelin(C18:1) & $1(0.82-1.25)$ & $1.02(0.82-1.19)$ & $1.19(0.91-1.49)$ & $1.35(1.08-1.54)$ & 0.002 & 0.01 & 0.21 & 0.17 \\
\hline Ceramide(C20:0) & $1(0.85-1.22)$ & $1.1(0.91-1.34)$ & $1.2(0.88-1.47)$ & $1.36(1.13-1.66)$ & 0.002 & 0.01 & 0.25 & 0.11 \\
\hline Ceramide(C18:1) & $1(0.86-1.18)$ & $1.19(0.9-1.45)$ & $0.93(0.76-1.26)$ & $1.35(1.1-1.66)$ & 0.002 & 0.01 & 0.49 & $8 \times 10^{-4}$ \\
\hline Ceramide(C22:0) & $0.99(0.81-1.2)$ & $1.25(0.91-1.53)$ & $1.22(1.02-1.43)$ & $1.41(1.18-1.73)$ & 0.002 & 0.01 & 0.21 & 0.17 \\
\hline Sphingomyelin(C18:0) & $1(0.84-1.2)$ & $0.96(0.78-1.16)$ & $1.11(0.89-1.33)$ & $1.28(1.09-1.46)$ & 0.01 & 0.03 & 0.16 & 0.31 \\
\hline Hexosylceramide(C18:0) & $1(0.87-1.16)$ & $0.88(0.69-1.17)$ & $1.29(1.1-1.58)$ & $1.14(0.96-1.41)$ & 0.01 & 0.03 & -0.09 & 0.56 \\
\hline Hexosylceramide(C24:1) & $1(0.76-1.26)$ & $0.87(0.66-1.05)$ & $1.17(0.93-1.47)$ & $0.99(0.75-1.2)$ & 0.01 & 0.04 & -0.11 & 0.48 \\
\hline Ceramide(C16:0) & $1(0.85-1.16)$ & $1.2(0.88-1.37)$ & $1.03(0.86-1.24)$ & $1.24(1.06-1.41)$ & 0.02 & 0.06 & 0.31 & 0.04 \\
\hline Dihydro-S1P(d18:0) & $1(0.82-1.22)$ & $0.88(0.77-1.07)$ & $1.05(0.83-1.3)$ & $1.23(1.03-1.52)$ & 0.02 & 0.06 & 0.21 & 0.18 \\
\hline Ceramide(C24:1) & $1(0.88-1.11)$ & $1.14(0.85-1.36)$ & $1.01(0.89-1.21)$ & $1.17(1.06-1.38)$ & 0.03 & 0.07 & 0.25 & 0.10 \\
\hline Ceramide(C24:0) & $1(0.84-1.17)$ & $1.15(0.93-1.45)$ & $1.14(0.94-1.36)$ & $1.34(1.08-1.67)$ & 0.04 & 0.10 & 0.15 & 0.35 \\
\hline Sphingosine(d18:1) & $1(0.67-1.41)$ & $1.04(0.67-1.57)$ & $1.04(0.73-1.58)$ & $1.32(0.96-1.93)$ & 0.05 & 0.11 & 0.11 & 0.49 \\
\hline S1P(d18:1) & $1(0.85-1.18)$ & $0.94(0.77-1.11)$ & $1.03(0.9-1.22)$ & $1.11(1-1.31)$ & 0.07 & 0.13 & 0.08 & 0.60 \\
\hline Sphingomyelin(C12:0) & $0.99(0.76-1.31)$ & $0.92(0.73-1.25)$ & $0.89(0.6-1.35)$ & $1.2(1-1.6)$ & 0.09 & 0.16 & 0.35 & 0.02 \\
\hline Hexosylceramide(C16:0) & 1 (0.88-1.15) & $0.88(0.71-1.05)$ & $1.11(1-1.28)$ & $0.96(0.81-1.26)$ & 0.10 & 0.18 & -0.07 & 0.65 \\
\hline \multicolumn{9}{|l|}{ Fatty acids } \\
\hline Oleic acid & $1(0.79-1.21)$ & $1.2(0.94-1.8)$ & $1.13(0.83-1.47)$ & $1.77(1.26-2.09)$ & 0.002 & 0.022 & 0.26 & 0.08 \\
\hline Palmitoleic acid & $1(0.68-1.4)$ & $1.25(0.64-2.22)$ & $1.05(0.6-1.84)$ & $2(1.3-3.25)$ & 0.01 & 0.04 & 0.30 & 0.05 \\
\hline Palmitic acid & $1(0.76-1.32)$ & $1.1(0.8-1.45)$ & $1.1(0.86-1.5)$ & $1.5(1.05-1.9)$ & 0.01 & 0.05 & 0.25 & 0.11 \\
\hline$\alpha$-Linolenic acid & $1(0.73-1.37)$ & $1.3(0.97-1.62)$ & $1.02(0.76-1.3)$ & $1.32(0.97-1.95)$ & 0.02 & 0.07 & 0.21 & 0.18 \\
\hline Myristoic acid & $1(0.6-1.7)$ & $1.26(0.53-2.39)$ & $0.83(0.46-1.43)$ & $1.7(1.07-2.5)$ & 0.03 & 0.07 & 0.31 & 0.04 \\
\hline Dihomo- $\gamma$-linolenic acid & $1(0.79-1.23)$ & $1.34(0.96-2.05)$ & $1.22(0.96-1.4)$ & $1.61(1.21-2.12)$ & 0.03 & 0.07 & 0.34 & 0.02 \\
\hline \multicolumn{9}{|l|}{ Eicosanoids } \\
\hline $\mathrm{LTE}_{4}$ & $1(0.5-1.89)$ & $1.35(0.78-3.96)$ & $3.48(1.65-6.1)$ & $2.1(1.1-5.18)$ & 0.009 & 0.33 & 0.15 & 0.34 \\
\hline 14,15-DiHETE & $1(0.71-1.53)$ & $0.74(0.46-1.08)$ & $0.64(0.42-0.99)$ & $0.71(0.49-0.97)$ & 0.03 & 0.63 & 0.24 & 0.12 \\
\hline 19,20-DiHDPA & $1(0.66-1.56)$ & $0.73(0.47-1.17)$ & $0.75(0.5-1.1)$ & $0.63(0.42-0.93)$ & 0.05 & 0.63 & -0.11 & 0.46 \\
\hline 8-HEPE & $1(0.71-1.41)$ & $0.45(0.3-0.82)$ & $0.82(0.39-1.42)$ & $0.95(0.52-1.57)$ & 0.07 & 0.64 & 0.04 & 0.81 \\
\hline
\end{tabular}

Data are presented as median ( $95 \% \mathrm{CI}$ ). Displayed data were selected based upon $\mathrm{p}<0.1$. The full list of all detected metabolites is provided in supplementary table E4, (sphingolipids), supplementary table E5 (fatty acids) and supplementary table E6 (eicosanoids). Median fold change and confidence intervals were estimated using 500 iterations of bootstrap resampling. ICS: inhaled corticosteroids; S1P: sphingosine-1-phosphate; LTE 4 : cysteinyl leukotriene $E_{4}$. 
high dietary intake of oleic acid has been reported to associate with asthma [30]. Taken together, our findings support increasing evidence that diet and the gut microbiome health play a role in asthma [31] and suggest that the association is more prominent in the steroid-naïve mild asthma group.

The moderate and severe asthma groups were characterised by a number of differentially abundant metabolites. Of particular interest were the increases in S1P(d18:1), OEA and N-palmitoyltaurine (table 2), which strongly contributed to the separation of patients with moderate/severe asthma from controls (CV1; figure $3 \mathrm{~b}$ ), and represented major nodes of metabolite clustering (figure 2). Importantly, the shifts appear to be steroid independent, with no correlation with ICS or OCS observed. This suggests that these shifts in metabolites are a consequence of processes intrinsic to the disease itself. The lipid mediator $\mathrm{S} 1 \mathrm{P}(\mathrm{d} 18: 1)$, which activates the G-protein coupled receptors (GPCRs), has been well characterised for its multiple roles in pulmonary disease, including cell migration, modulating endothelial and epithelial barriers, and upregulating inflammatory metabolic pathways [32], and in the activation of vagal nerve transient receptor potential vanilloid type 1 (TRPV1) receptors in a murine asthma model [33]. Likewise, OEA and $\mathrm{N}$-acyltaurines can also activate TRPV1 receptors $[34,35]$. TRPV1 receptors act as $\mathrm{Ca}^{2+}$ ion channels in sensory nerves, contributing to chemotaxis, bronchoconstriction, mucous secretion, airway irritation and the urge to cough $[36,37]$. Recently, methylthioadenosine (also contributing uniquely to CV1) was found to signal through the GPCR adenosine receptor A2B (A2BAR), activating the protein kinase C cascade [38] and, therefore, potentially TRPV1 [39]. A2BAR is present on sensory nerves [40] and is implicated in bronchoconstriction and cytokine release [41]. It is therefore plausible that the increases we observed in S1P (d18:1), OEA, N-palmitoyltaurine and methylthioadenosine could enhance TRPV1 and A2BAR signalling in asthma; however, further direct evidence will be required to test this hypothesis.

Metabolites that correlated with ICS dose exhibited the greatest difference between the groups studied using both univariate and multivariate statistics (table 2 and figure 3); these differences were most pronounced in the OCS-treated individuals (figure 1). This suggests that the association between metabolic profile and ICS treatment is greater than that between metabolic profile and disease, underscoring the potential physiological impact of treatment. ICS are considered to be relatively safe at low-moderate doses [42]; our findings did indeed indicate that higher doses (and oral treatment) are associated with the most pronounced metabolic effects. Reduced levels of DHEA-S, cortisone and cortisol reflect hypothalamuspituitary-adrenal axis suppression, a well-documented consequence of high-dose ICS treatment [42, 43]. Comhair et al. [12] also found suppression of plasma steroids in patients with severe asthma in a metabolomics study. To our knowledge, however, reduced ProHyp and pipecolic acid have not been reported previously in association with therapeutic corticosteroids administration. ProHyp is a dipeptide predicted to play a role in bone health and turnover [44]. Following oral administration in rats, ProHyp has been found in bone marrow osteoblasts and stems cells, as well as in chondrocytes, synovial cells and osteoclasts. Additionally, both ProHyp and pipecolic acid have been found to be significantly dysregulated in post-menopausal women with bone disease [45]. Although the mechanisms leading to their dysregulation remain unclear, we postulate that reduced ProHyp and pipecolic acid could reflect the increased risk of osteoporosis and bone injury associated with corticosteroid treatment [46]. Furthermore, because all individuals with moderate or severe asthma were treated with corticosteroids, we are unable to distinguish, with certainty, between the effects of disease severity and treatment. Additional studies in steroid-naïve cohorts will be necessary to provide further clarity.

There are a number of study limitations that could affect the reported findings. Corticosteroid metabolites were not quantified in vivo; rather, we relied on patient reporting and clinical records to assess the metabolic changes associated with corticosteroid treatment. Furthermore, our relatively small study lacks a validation cohort and is not well matched demographically (e.g. age, body mass index, gender). In addition, the identification of metabolites was based on an in-house database of chemical standards. It is distinctly plausible that metabolites not included in the database could be of importance in distinguishing patients with mild-to-moderate asthma from healthy individuals. Accordingly, while the current findings show a modest underlying metabolic shift in the circulation in asthmatic individuals that is dependent upon disease severity, there is a distinct need to further analyse these metabolic patterns in a larger cohort using a more comprehensive metabolite database. The observed shifts in mild asthma are largely associated with exogenous metabolites, while those in moderate and severe asthma are potentially associated with activation of GPCRs and TRPV1. Importantly, metabolic changes associated with corticosteroid treatment were clearly evident in the metabolome, even with moderate ICS dose alone, and could reflect increased osteoporosis risk. These findings highlight the need to further investigate the metabolic effects of both ICS and OCS treatment in individuals with asthma.

\section{Acknowledgements}

The authors wish to thank David Broadhurst for donating the QC-RSC algorithm and for providing statistical support, as well as Åsa Wheelock for performing the post hoc power calculation. 


\section{References}

1 Global Initiative for Asthma (GINA). Global strategy for asthma management and prevention. 2012. www. ginasthma.org Date last accessed: 5 September 2013.

2 Gauthier M, Ray A, Wenzel SE. Evolving concepts of asthma. Am J Respir Crit Care Med 2015; 192: 660-668.

3 Anderson GP. Endotyping asthma: new insights into key pathogenic mechanisms in a complex. heterogeneous disease. Lancet 2008; 372: 1107-1119.

4 Chung KF, Wenzel SE, Brozek JL, et al. International ERS/ATS guidelines on definition, evaluation and treatment of severe asthma. Eur Respir J 2014; 43: 343-373.

5 Dunn WB, Broadhurst DI, Atherton HJ, et al. Systems level studies of mammalian metabolomes: the roles of mass spectrometry and nuclear magnetic resonance spectroscopy. Chem Soc Rev 2011; 40: 387-426.

6 Broadhurst DI, Kell DB. Statistical strategies for avoiding false discoveries in metabolomics and related experiments. Metabolomics 2006; 2: 171-196.

7 Holmes TH. Ten categories of statistical errors: a guide for research in endocrinology and metabolism. Am J Physiol Endocrinol Metab 2004; 286: E495-E501.

8 Jung J, Kim SH, Lee HS, et al. Serum metabolomics reveals pathways and biomarkers associated with asthma pathogenesis. Clin Exp Allergy 2013; 43: 425-433.

9 Ried JS, Baurecht H, Stuckler F, et al. Integrative genetic and metabolite profiling analysis suggests altered phosphatidylcholine metabolism in asthma. Allergy 2013; 68: 629-636.

10 Loureiro CC, Duarte IF, Gomes J, et al. Urinary metabolomic changes as a predictive biomarker of asthma exacerbation. J Allergy Clin Immunol 2014; 133: 261-263.

11 Saude EJ, Skappak CD, Regush S, et al. Metabolomic profiling of asthma: diagnostic utility of urine nuclear magnetic resonance spectroscopy. J Allergy Clin Immunol 2011; 127(3): 757-764.

12 Comhair SA, McDunn J, Bennett C, et al. Metabolomic endotype of asthma. J Immunol 2015; 195: 643-650.

13 Hinks TS, Zhou X, Staples KJ, et al. Innate and adaptive T cells in asthmatic patients: relationship to severity and disease mechanisms. J Allergy Clin Immunol 2015; 136: 323-333.

14 Vijayanand P, Seumois G, Pickard C, et al. Invariant natural killer T cells in asthma and chronic obstructive pulmonary disease. N Engl J Med 2007; 356: 1410-1422.

15 Dunn WB, Broadhurst D, Begley P, et al. Human serum metabolome C. Procedures for large-scale metabolic profiling of serum and plasma using gas chromatography and liquid chromatography coupled to mass spectrometry. Nat Protoc 2011; 6: 1060-1083.

16 Kirwan JA, Broadhurst DI, Davidson RL, et al. Characterising and correcting batch variation in an automated direct infusion mass spectrometry (DIMS) metabolomics workflow. Anal Bioanal Chem 2013; 405: 5147-5157.

17 Hastie T, Tibshirani R, Friedman J. The Elements of Statistical Learning: Data Mining, Inference, and Prediction. 2nd Edn. New York, Springer, 2009.

18 Hollywood KA, Winder CL, Dunn WB, et al. Exploring the mode of action of dithranol therapy for psoriasis: a metabolomic analysis using HaCaT cells. Mol Biosyst 2015; 11: 2198-2209.

19 Krzanowski WJ. Principles of Multivariate Analysis: A User’s Perspective. Oxford, Oxford University Press, 1988.

20 Troyanskaya O, Cantor M, Sherlock G, et al. Missing value estimation methods for DNA microarrays. Bioinformatics 2001; 17: 520-525.

21 Peres-Neto PR, Jackson DA, Somers KM. Giving meaningful interpretation to ordination axes: assessing loading significance in principal component analysis. Ecology 2003; 84: 2347-2363.

22 Levy BD. Sphingolipids and susceptibility to asthma. N Engl J Med 2013; 369: 976-978.

23 McGeachie MJ, Dahlin A, Qiu W, et al. The metabolomics of asthma control: a promising link between genetics and disease. Immun Inflamm Dis 2015; 3: 224-238.

24 Nikolaizik WH, Marchant JL, Preece MA, et al. Nocturnal cortisol secretion in healthy adults before and after inhalation of budesonide. Am J Respir Crit Care Med 1996; 153: 97-101.

25 Clark DJ, Grove A, Cargill RI, et al. Comparative adrenal suppression with inhaled budesonide and fluticasone propionate in adult asthmatic patients. Thorax 1996; 51: 262-266.

26 Daynes RA, Jones DC. Emerging roles of PPARs in inflammation and immunity. Nat Rev Immunol 2002; 2: 748-759.

27 Yeh YY, Liu L. Cholesterol-lowering effect of garlic extracts and organosulfur compounds; human and animal studies. J Nutr 2001; 131: Suppl. 3, 989S-993S.

28 Jalal MAF, Collin HA. Estimation of caffeine, theophylline, and theobromine in plant material. New Phytol 1975; 76: $277-281$.

29 Li M, Wang B, Zhang M, et al. Symbiotic gut microbes modulate human metabolic phenotypes. Proc Natl Acad Sci USA 2008; 105: 2117-2122.

30 Nagel G, Linseisen J. Dietary intake of fatty acids. antioxidants and selected food groups and asthma in adults. Eur J Clin Nutr 2005; 59: 8-15.

31 Huang YJ, Boushey HA. The microbiome in asthma. J Allergy Clin Immunol 2015; 135: 25-30.

32 Brinkmann V, Baumruker T. Pulmonary and vascular pharmacology of sphingosine 1-phosphate. Curr Opin Pharmacol 2006; 6: 244-250.

33 Trankner D, Hahne N, Sugino K, et al. Population of sensory neurons essential for asthmatic hyperreactivity of inflamed airways. Proc Natl Acad Sci USA 2014; 111: 11515-11520.

34 Wang X, Miyares RL, Ahern GP. Oleoylethanolamide excites vagal sensory neurones. induces visceral pain and reduces short-term food intake in mice via capsaicin receptor TRPV1. J Physiol 2005; 564(Pt 2): 541-547.

35 Saghatelian A, McKinney MK, Bandell M, et al. A FAAH-regulated class of N-acyl taurines that activates TRP ion channels. Biochemistry 2006; 45: 9007-9015.

36 Lee LY, Gu Q. Role of TRPV1 in inflammation-induced airway hypersensitivity. Curr Opin Pharmacol 2009; 9: 243-249.

37 McGarvey LP, Butler CA, Stokesberry S, et al. Increased expression of bronchial epithelial transient receptor potential vanilloid 1 channels in patients with severe asthma. J Allergy Clin Immunol 2014; 133: 704-712 e704.

38 Limm K, Wallner S, Milenkovic VM, et al. The metabolite 5'-methylthioadenosine signals through the adenosine receptor A2B in melanoma. Eur J Cancer 2014; 50: 2714-2724.

39 Szallasi A, Cortright DN, Blum CA, et al. The vanilloid receptor TRPV1: 10 years from channel cloning to antagonist proof-of-concept. Nat Rev Drug Discov 2007; 6: 357-372. 
40 Burnstock G, Sawynok J. Adenosine triphosphate and adenosine receptors and pain. In: Beaulieu P, Lussier D, Porreca F, Dickenson A, eds. Pharmacology of Pain. Seattle, IASP Press, 2010; pp. 303-326.

41 Aherne CM, Kewley EM, Eltzschig HK. The resurgence of A2B adenosine receptor signaling. Biochim Biophys Acta 2011; 1808: 1329-1339.

42 Raissy HH, Kelly HW, Harkins M, et al. Inhaled corticosteroids in lung diseases. Am J Respir Crit Care Med 2013; 187: 798-803.

43 Kannisto S, Laatikainen A, Taivainen A, et al. Serum dehydroepiandrosterone sulfate concentration as an indicator of adrenocortical suppression during inhaled steroid therapy in adult asthmatic patients. Eur J Endocrinol 2004; 150: 687-690.

44 Husek P, Svagera Z, Vsiansky F, et al. Prolyl-hydroxyproline dipeptide in non-hydrolyzed morning urine and its value in postmenopausal osteoporosis. Clin Chem Lab Med 2008; 46: 1391-1397.

45 Moayyeri A, Hart D, Erte I, et al. Causal metabolomic pathways to osteoporosis in elderly women. In: European Calcified Tissue Society Congress; 18-21 May 2013; Lisbon, Portugal. Bone Abstracts 2013; 1: OC3.6.

46 Wong CA, Walsh LJ, Smith CJ, et al. Inhaled corticosteroid use and bone-mineral density in patients with asthma. Lancet 2000; 355: 1399-1403. 